\title{
Los estudios métricos de información en el contexto de la pandemia por covid-19
}

\author{
Information metric studies in the context of the covid-19 pandemic \\ Os estudos métricos da informação no contexto da pandemia por \\ covid-19
}

\author{
Nancy Sánchez-Tarragó ${ }^{1, a}$ \\ nancy.sanchez@ufrn.br | https://orcid.org/o000-0002-5114-6072 \\ Grisel Zacca González ${ }^{2, a}$ \\ grisel.zacca@infomed.sld.cu | https://orcid.org/0000-0003-4670-5092 \\ ${ }^{1}$ Universidad Federal de Rio Grande do Norte, Departamento de Ciencia de la Información. Natal, RN, Brasil. \\ ${ }^{2}$ Centro Nacional de Información de Ciencias Médicas. La Habana, LH, Cuba. \\ a Doctorado en Información Científica y Documentación por la Universidad de Granada, España.
}

\section{RESUMEN}

Esta nota expone un breve análisis coyuntural de la emergencia de salud pública por covid-19 y algunos de los temas más relevantes relacionados con la información y comunicación en salud. De esta manera, se pretende contextualizar la aplicación de los estudios métricos de información como herramientas de análisis de realidades complejas y multidimensionales.

Palabras clave: Estudios métricos de la información; Covid-19; Información científica; Desinformación; Salud Pública.

\section{RESUMO}

Esta nota de conjuntura apresenta uma breve análise da emergência de saúde pública devido à covid-19 e algumas das questões mais relevantes relacionadas à informação e comunicação em saúde. Desta forma, pretende-se contextualizar a aplicação de estudos métricos de informação como ferramentas de análise de realidades complexas e multidimensionais.

Palavras-chave: Estudos métricos da informação; Covid-19; Informação científica; Desinformação; Saúde Pública. 
Reciis - Revista Eletrônica de Comunicação, Informação \& Inovação em Saúde, Rio de Janeiro, v. 15, n. 4, p. 801-807, out./dez. 2021 [www.reciis.icict.fiocruz.br] e-ISSN 1981-6278

\section{ABSTRACT}

This conjuncture note presents a brief analysis of the public health emergency due to covid-19 and some of the most relevant issues related to health information and communication. In this way, it intends to contextualize the application of information metric studies as tools for the analysis of complex and multidimensional realities.

Keyword: Metric studies of information; Covid-19; Scientific information; Disinformation; Public Health.

\section{Contribuciones del autor:}

Diseño de estudio: Nancy Sánchez-Tarragó y Grisel Zacca González.

Adquisición, análisis o interpretación de datos: Nancy Sánchez-Tarragó y Grisel Zacca González.

Redacción del manuscrito: Nancy Sánchez-Tarragó y Grisel Zacca González.

Revisión crítica del contenido intelectual: Nancy Sánchez-Tarragó y Grisel Zacca González.

Declaracion de conflicto de interes: no hay

Fuente de financiamiento: no hay.

Historial del artículo: enviado: 25 oct. 2021 | aceptado: 25 oct. 2021 | publicado: 10 nov. 2021.

Presentación anterior: no hay.

Licencia CC BY-NC atribución nocomercial. Con esta licencia se permite acceder, descargar, copiar, imprimir, compartir, reutilizar y distribuir los artículos, siempre que sean para uso no comercial y con la citación de la fuente, otorgando los debidos créditos de autoría y mención a Reciis. En tales casos, no se requiere permiso de los autores o editores. 


\section{INTRODUCCIÓN}

Los estudios métricos de la información se han desarrollado al unísono con el avance de la ciencia y, en los últimos años, han ganado aceptación por su utilidad en la descripción, evaluación y monitoreo de la actividad científica y de los flujos de información, en general. Se complementan y se alimentan de otras disciplinas que estudian la ciencia y la sociedad como la economía, la sociología, la filosofía y la historia. Entre sus principales propósitos están comprender la dinámica de la ciencia, identificar nuevos frentes de investigación, caracterizar la estructura intelectual de un dominio de conocimiento y sus patrones de comunicación, analizar redes temáticas y de colaboración, evaluar resultados de investigación, contribuir con prospecciones y con la definición de políticas científicas. La bibliometría, la cienciometría, la informetría y, recientemente, la altmetría (altmetrics), son los tipos de estudios métricos más utilizados en la actualidad. Para contextualizar algunos de sus usos en la salud, esta nota presenta un breve análisis coyuntural de la emergencia de salud pública por covid-19 y temas relevantes relacionados con la información y comunicación en salud.

\section{INFORMACIÓN Y COMUNICACIÓN PARA ENFRENTAR LA COVID-19 EN UN CONTEXTO DE DESIGUALDADES Y DESINFORMACIÓN}

Cuando a inicios de 2020 se conoció que una nueva enfermedad respiratoria causada por un virus hasta entonces desconocido se alastraba por el territorio chino y países asiáticos, nadie imaginaba que pocas semanas después todos los países del orbe serian afectados por uno de los mayores desastres sanitarios, económicos y sociales de las últimas centurias. En 2021, tras varias olas de casos de covid-19, se acumulan más de 240 millones de casos y casi 5 millones de fallecidos (WHO, 2021). La crisis sanitaria, económica y social provocada por la pandemia ha sido realmente, en palabras de Boaventura de Souza Santos, apenas un agravamiento de la 'crisis' perpetua que vive el mundo, fundamentalmente desde la década de 1980, a medida que el neoliberalismo se ha impuesto como la versión dominante del capitalismo y ha sometido al mundo a la lógica del sector financiero global (SANTOS, 2020). Es una crisis que oculta la escandalosa concentración de riqueza y desigualdad social, pero que justifica los cortes en políticas públicas relacionados con la ciencia, la educación, la salud y la seguridad social, que se mostraron cruciales e insuficientes durante la pandemia.

Mujeres, indígenas, afrodescendientes, población con menores niveles educativos, inmigrantes y refugiados, son los grupos más afectados, que más sintieron y sienten los efectos perversos de la desigualdad en tiempos de pandemia de covid-19. Estas desigualdades se observan también a nivel de países y regiones geopolíticas, con marcadas asimetrías en el acceso a capacidades y recursos para enfrentar la pandemia, ya sea máscaras, productos de higiene, respiradores, insumos, vacunas, tecnologías de información, literatura científica, capacidades para investigación científica y publicación, y competencias y recursos para filtrar o chequear la veracidad de las informaciones.

Desde las primeras semanas de 2020, el volumen y ritmo de producción de información científica relacionada con covid-19 han sido enormes. Centenas de estudios métricos han sido realizados desde las diferentes regiones del mundo, utilizando diversas bases o conjuntos de datos y variados indicadores para analizar esta dinámica. Se estima que por lo menos $4 \%$ de la producción científica de 2020 en la base de datos Dimensions fue dedicada a covid-19, lo que corresponde a más de 200000 artículos hasta diciembre de 2020 (ELSE, 2020). La emergencia de covid-19 también colocó en jaque el acceso a la información y a la educación, evidenciando, una vez más, la insoslayable necesidad de una ciencia abierta, transparente y colaborativa, tanto para acelerar el desarrollo de vacunas y tratamientos, como para preparar a los profesionales de salud y proporcionar al público información de calidad sobre las medidas de salud pública. 
De hecho, una de las más importantes transformaciones en la comunicación científica, catalizada por la emergencia de covid-19, ha sido la generalización de los preprint como vía expedita para la comunicación en acceso abierto de resultados científicos. Más de 30 ooo artículos sobre covid-19 publicados en 2020 fueron preprints (entre 17\% y 30\% del total de artículos según la base de datos analizada) (ELSE, 2020). Otras iniciativas han estado relacionadas con la implantación de observatorios, directorios de fuentes de información, portales de revistas y guías de evidencias clínicas.

No obstante, el enorme volumen de artículos y preprints publicados a gran velocidad, representa desafíos para la propia integridad, calidad y credibilidad de la ciencia. El sitio Retraction Watch lista 181 artículos (RETRACTED..., 2021) sobre covid-19 (incluyendo preprints) retractados o retirados por diversos motivos, desde fallas metodológicas y problemas con los datos primarios hasta fraude. La ciencia 'defectuosa' que contienen estos trabajos muchas veces se ha hecho viral (son trabajos muy descargados, citados y compartidos en redes sociales aun después de retractados) y ha alimentado cadenas de desinformación, sobre todo cuando se refieren a temas muy polémicos y politizados como el posible origen no natural del virus y la efectividad de medicamentos como la hidroxicloroquina (LÓPEZ-CÓZAR; MARTIN-MARTIN, 2020; SANTOS-D’AMORIM; MELO; SANTOS, 2021).

La infodemia que acompaña a la pandemia desde sus primeros días no es un fenómeno originado con la covid-19 pero sin dudas se ha amplificado por la penetración global de Internet y las redes sociales, que modifican las formas de interacción con la información. Tres características de este nuevo escenario informacional merecen ser destacadas: un volumen cada vez mayor de información circula en tiempo real entre redes de confianza (por ejemplo, en el grupo familiar de WhatsApp) lo que hace menos probable que la veracidad de estos mensajes sea cuestionada; aumento de la velocidad de difusión de información por el uso generalizado de celulares y smartphone; y un debilitamiento de la credibilidad en los hechos objetivos a favor de una glorificación de la opinión basada en sentimientos y creencias (WARDLE; DERAKHSHAN, 2017). En este régimen de posverdad las creencias y opiniones erguidas como 'verdades' o 'realidades alternativas' se corresponden a narrativas que, generalmente, buscan deliberadamente promover determinada percepción sobre la verdad para someter a los individuos e instituciones a relaciones de poder.

En este contexto de asimetría informacional, líderes religiosos y de gobierno, entre otros agentes, han utilizado su autoridad y poder para producir y propagar desinformación (teorías de la conspiración, noticias falsas, pseudociencia), alrededor de todas las aristas relacionadas con el virus y la enfermedad (LIMA et al., 2020). En redes sociales, robots (bots) y algoritmos de inteligencia artificial contribuyen a la reproducción de estas narrativas de control, generalmente sesgadas, y asociadas a ideologías de extrema-derecha y de negacionismo científico. Las consecuencias de esta pandemia de desinformación han sido trágicas, sobre todo porque figuras públicas, como los presidentes Donald Trump e Jair Bolsonaro, que deberían liderar los esfuerzos de articulación de medidas de salud pública y efectiva comunicación de estas a la población, han sido piezas centrales en el engranaje de producción y diseminación de desinformación, motivados por intereses políticos y económicos (CÉSAR; MACIEL, 2021; LIMA et al. 2020).

\section{LOS ESTUDIOS MÉTRICOS DE INFORMACIÓN Y LA COVID-19}

En este contexto, los estudios métricos han sido utilizados para analizar las dinámicas de producción de la literatura científica sobre covid-19 y también los flujos de información (y desinformación) en las redes sociales. En el primer caso, se trata de estudios bibliométricos y cienciométricos que han analizado la estructura intelectual de estos dominios con indicadores de producción, citación y relacionales, produciendo, por ejemplo, mapas de tópicos, áreas temáticas y líneas de investigación sobre covid-19. Esto ha permitido identificar los temas más investigados a nivel mundial: características genéticas y cepas de coronavirus, factores de riesgo y síntomas de la enfermedad, manejo clínico y medidas de salud pública (ARISTOVNIK; 
RAVŠELJ; UMEK, 2020; CHENG; CAO, LIAO, 2020; HERRERA-VIEDMA et al., 2020). Estados Unidos y China han sido identificados como los países más productivos en la temática (ZYOUD; AL-JABI, 2020; YU et al., 2020; BELLI et al., 2020). Por otra parte, se observa que la investigación sobre covid-19 se caracteriza por una interdisciplinariedad mayor que los estudios de epidemias/pandemias anteriores, con más de 200 dominios temáticos relacionados directa o indirectamente con la prevención, diagnóstico, tratamiento y respuesta social (ARENCIBIA-JORGE et al., 2020).

La producción científica latinoamericana sobre covid-19 también ha sido objeto de estudios bibliométricos. Brasil, México y Chile fueron identificados como los países más productivos en el estudio de Espinosa et al. (2021). Otros estudios han analizado, por ejemplo, las principales líneas de investigación en la región, apuntando para diagnóstico y tratamiento de covid-19 (ESPINOSA et al., 2021) y los servicios de salud, las políticas y medidas de salud pública (GALLEGOS et al., 2020).

Los estudios métricos también han constatado el agravamiento de desigualdades de género y socioeconómicas causadas por la pandemia, y sus impactos, por ejemplo, en la producción científica. Investigadoras mujeres han publicado menos y han tenido menos participación en la revisión por pares que sus pares masculinos, lo que evidencia mayores afectaciones de la covid-19 relacionadas con la sobrecarga emocional y de responsabilidades domésticas (MURIC; LERMAN; FERRARA, 2021; SQUAZONNI et al., 2021). En el caso de la productividad por país, Müller et al. (2020) encontraron marcadas diferencias geográficas y nacionales. Países con infraestructura científica estable, niveles previamente altos de productividad en publicaciones y sistemas bien organizados de atención médica consiguieron, no solo producir altas tasas de publicaciones sobre covid-19, sino también mantener la publicación sobre otras temáticas en niveles similares al periodo pre-pandémico.

Por otra parte, durante los últimos años, la Declaración de San Francisco y el Manifiesto de Leiden han enfatizado la importancia de utilizar métricas alternativas, como las basadas en interacciones en redes sociales, para substituir o complementar los tradicionales indicadores de impacto basados en citaciones, por sus conocidas limitaciones (HICKS et al., 2015). Estudios recientes han analizado las fortalezas y debilidades de la altmetria para identificar artículos relevantes y de calidad relacionados con covid-19 teniendo en cuenta la expansión cada vez mayor de las redes sociales para la divulgación de la ciencia (BOETTO et al., 2021; KHATTER et al., 2021).

Finalmente, los estudios métricos que analizan los flujos de información sobre covid-19 en redes sociales han sido muy importantes para caracterizar, por ejemplo, las dinámicas de la desinformación y su relación con disputas de poder e intereses económicos. Análisis de publicaciones e interacciones en Youtube, Facebook, Instagram y Twitter han mostrado las percepciones y sentimientos de los usuarios de estas redes acerca de la vacunación; las disputas y narrativas sobre hidroxicloroquina, ivermectina y azitromicina por parte de autoridades públicas para legitimar posturas populistas y neoliberales; la propagación de teorías de la conspiración, vinculadas a tramas geopolíticas y económicas; y el uso distorsionado de términos y conceptos médicos y científicos en las cadenas de desinformación, entre muchos otros temas (MONARI; SANTOS; SACRAMENTO, 2020; MACHADO et al., 2020; YOUSEFINAGHANI et al., 2021).

Sin dudas, los estudios métricos de información, en sus variadas tipologías y metodologías, contribuyen a iluminar fenómenos complejos como los asociados a la emergencia de covid-19, permitiendo una mejor comprensión de sus aristas científicas, sociales y políticas, y respuestas más acertadas por parte de gestores y académicos. 
Reciis - Revista Eletrônica de Comunicação, Informação \& Inovação em Saúde, Rio de Janeiro, v. 15, n. 4, p. 801-807, out./dez. 2021 [www.reciis.icict.fiocruz.br] e-ISSN 1981-6278

\section{REFERENCIAS}

ARENCIBIA-JORGE, Ricardo et al. The multidisciplinary nature of covid-19 research. Iberoamerican Journal of Science Measurement and Communication, Hong Kong, v. 1, n. 1, p. 1-11, 2020. DOI: https:// doi.org/10.47909/ijsmc.13. Disponible en: https://pub.colnes.org/index.php/ijsmc/article/view/13. Acceso en: 20 oct. 2021.

ARISTOVNIK, Aleksander; RAVŠELJ, Dejan; UMEK, Lan. Bibliometric analysis of Covid-19 across science and social science research landscape. Sustainability, Basel, v. 12, n. 21, p. 9132, 2020. DOI: https://doi. org/10.3390/su12219132. Disponible en: https://www.mdpi.com/2071-1050/12/21/9132. Acceso en: 20 oct. 2021.

BELLI, Simone et al. Coronavirus mapping in scientific publications: When science advances rapidly and collectively, is access to this knowledge open to society? Scientometrics, Basel, v. 124, p. 26612685, 2020. DOI: https://doi.org/10.1007/s11192-020-03590-7. Disponible en: https://link.springer.com/ article/10.1007\%2Fs11192-020-03590-7. Acceso en: 20 oct. 2021.

BOETTO, Erik et al. Using altmetrics for detecting impactful research in quasi-zero-day time-windows: the case of covid-19. Scientometrics, Amsterdam, v. 126, n. 2, p. 1189-1215, 2021. DOI: https://doi.org/10.1007/ s11192-020-03809-7. Disponible en: https://link.springer.com/article/10.1007\%2Fs11192-020-03809-7. Acceso en: 20 oct. 2021.

CEZAR, Lilian Sagio; MACIEL, Anderson Jamar Neves. Infodemia no contexto da pandemia de COVID-19 no Brasil: uma política de contaminação?. Liinc em Revista, Rio de Janeiro, v. 17, n. 1, p. e5703, 2021. Disponible en: http://revista.ibict.br/liinc/article/view/5703. Acceso en: 20 out. 2021.

CHENG, Xian; CAO, Qiang; LIAO, Stephen Shaoyi. An overview of literature on covid-19, Mers and Sars using text mining and latent Dirichlet allocation. Journal of Information Science, London, p. 1-17, sept. 2020. DOI: https://doi.org/10.1177/0165551520954674. Disponible en: https://journals.sagepub.com/doi/ full/10.1177/0165551520954674. Acceso en: 20 oct. 2021

ELSE, Holly. How a torrent of covid science changed research publishing - in seven charts. Nature, London, v. 588, n. 7839, p. 553-553, 2020. DOI: https://doi.org/10.1038/d41586-020-03564-y. Disponible en: https:// www.nature.com/articles/d41586-020-03564-y. Acceso en : 20 oct. 2021.

ESPINOSA, Isabel et al. A bibliometric analysis of covid-19 research in Latin America and the Caribbean. Revista de la Facultad de Medicina, Quito, v. 69, n. 3, p. 94520, 2021. DOI: https://doi.org/10.15446/ revfacmed.v69n3.94520. Disponible en: https://revistas.unal.edu.co/index.php/revfacmed/article/view/94520. Acceso en: 20 oct. 2021.

GALLEGOS, Miguel et al. Covid-19 in Latin America: a bibliometric analysis of scientific publications in health. Electronic Journal of General Medicine, Eastbourne, v. 17, n. 6, p. em261, 2020. https://doi.org/10.29333/ ejgm/8460. Disponible en: https://www.ejgm.co.uk/article/covid-19-in-latin-america-a-bibliometric-analysis-ofscientific-publications-in-health-8460. Acesso en: 20 oct. 2021.

HERRERA-VIEDMA, Enrique et al. Global trends in coronavirus research at the time of covid-19: A general bibliometric approach and content analysis using SciMAT. Profesional de la Información, León, v. 29, n. 3, e290322, 2020. Disponible en: https://revista.profesionaldelainformacion.com/index.php/EPI/article/view/ epi.2020.may.22. Acceso en: 20 oct. 2021.

HICKS, Diana et al. Bibliometrics: the Leiden Manifesto for research metrics. Nature, London, v. 520, n. 7548, p. 429-431, 2015. DOI: https://doi.org/10.1038/520429a. Disponible en: https://www.nature.com/ articles/520429a. Acceso en: 20 oct. 2021.

KHATTER, Amandeep et al. Is rapid scientific publication also high quality? Bibliometric analysis of highly disseminated covid-19 research papers. Learned Publishing, [s. I.], v. 34, n. 4, p. 568-577, 2021. DOI: https:// doi.org/10.1002/leap.1403. Disponible en: https://onlinelibrary.wiley.com/doi/10.1002/leap.1403. Acceso en: 20 oct. 2021.

LIMA, Clóvis Ricardo Montenegro de et al. Emergência de saúde pública global por pandemia de COVID-19: desinformação, assimetria de informações e validação discursiva. Folha de Rosto, Juazeiro do Norte, v. 6, n. 2, p. 5-21, 2020. DOI: https://doi.org/10.46902/2020n2p5-21. Disponible en: https://periodicos.ufca.edu.br/ojs/ index.php/folhaderosto/article/view/490. Acceso en: 20 out. 2021. 
LÓPEZ-CÓZAR, Emilio Delgado; MARTÍN-MARTÍN, Alberto. La viralidad de la ciencia defectuosa: el contagioso impacto mediático de un preprint en bioRxiv sobre el coronavirus y sus efectos en la comunicación científica. Granada: [s. n.], 2020. Disponible en: https://digibug.ugr.es/bitstream/handle/10481/60872/La\%20 viralidad $\% 20$ de $\% 20$ la $\% 20$ ciencia $\% 20$ defectuosa $\% 20$ el $\% 20$ contagioso $\% 20$ efecto $\% 20$ medi $\%$ C3\%A 1 tico $\% 20$ de\%20un\%20preprint $\% 20$ en $\% 20$ bioRxiv $\% 20$ sobre $\% 20$ el\%20coronavirus.pdf? sequence=1\&isAllowed=y. Acceso en: 20 out. 2021

MACHADO, Caio C. Vieira et al. Scientific (self) isolation. [S. I.]: Centro de Análise da Liberdade e do Autoritarismo, 2020. Disponible en: https://laut.org.br/wp-content/uploads/2020/11/Political-Self-Isolation-vF.pdf. Acceso en: 20 out. 2021.

MONARI, Ana Carolina Pontalti; SANTOS, Allan; SACRAMENTO, Igor. Covid-19 and (hydroxy)chloroquine: a dispute over scientific truth during Bolsonaro's weekly Facebook live streams. Journal of Science Communication, Trieste, v. 19, n. 7, p. A03, 2020. DOI: https://doi.org/10.22323/2.19070203. Disponible en: https://jcom.sissa.it/archive/19/07/JCOM_1907_2020_A03. Acceso en: 20 out. 2021.

MÜLLER, Simon M. et al. National publication productivity during the covid-19 pandemic: a preliminary exploratory analysis of the 30 countries most affected. Biology, Basel, v. 9, n. 9, p. 271, 2020. DOI: https:// dx.doi.org/10.3390\%2Fbiology9090271. Disponible en: https://www.mdpi.com/2079-7737/9/9/271. Acceso en: 20 out. 2021.

MURIC, Goran; LERMAN, Kristina; FERRARA, Emilio. Gender disparity in the authorship of biomedical research publications during the covid-19 pandemic: retrospective observational study. Journal of Medical Internet Research, Toronto, v. 23, n. 4, p. e25379, 2021. DOI: https://doi.org/10.2196/25379. Disponible en: https://www.jmir.org/2021/4/e25379. Acceso en: 20 out. 2021.

RETRACTED CORONAVIRUS (COVID-19) PAPERS. In: MARCUS, Adam; ORASNKY, Ivan. Retraction Watch. [S. I.], 2021. Disponible en: https://retractionwatch.com/retracted-coronavirus-covid-19-papers/. Acceso en: 23 oct. 2021

SANTOS, Boaventura de Sousa. A cruel pedagogia do vírus. Coimbra: Almedina, 2020.

SANTOS-D'AMORIM, Karen; MELO, Rinaldo Ribeiro de; SANTOS, Raimundo Nonato Macedo dos. Retractions and post-retraction citations in the covid-19 infodemic: is Academia spreading misinformation? Liinc em Revista, Rio de Janeiro, v. 17, n. 1, p. e5593-e5593, 2021. DOI: https://doi.org/10.18617/liinc. v17i1.5593. Disponible en: http://revista.ibict.br/liinc/article/view/5593. Acceso en: 20 out. 2021.

SQUAZZONI, Flaminio et al. Gender gap in journal submissions and peer review during the first wave of the covid-19 pandemic: a study on 2329 Elsevier journals. PLoS One, San Francisco, v. 16, n. 10, p. e0257919, 2021. DOI: https://doi.org/10.1371/journal.pone.0257919. Disponible en: https://journals.plos.org/plosone/ article?id=10.1371/journal. pone.0257919. Acceso en: 20 out. 2021.

WARDLE, Claire; DERAKHSHAN, Hossein. Information disorder: toward an interdisciplinary framework for research and policymaking. Strasbourg Cedex: Council of Europe, 2017. Disponible en: https://edoc.coe.int/en/ media/7495-information-disorder-toward-an-interdisciplinary-framework-for-research-and-policy-making.html. Acceso en: 20 out. 2021.

WORLD HEALTH ORGANIZATION (WHO). WHO Coronavirus (COVID-19) Dashboard. Genebra: La Organización, 2021. Disponible en: https://covid19.who.int/. Acceso en: 20 oct. 2021.

YOUSEFINAGHANI, Samira et al. An analysis of covid-19 vaccine sentiments and opinions on Twitter. International Journal of Infectious Diseases, Hamilton, v. 108, p. 256-262, 2021. DOI: https://doi. org/10.1016/j.ijid.2021.05.059. Disponible en: https://www.ijidonline.com/article/S1201-9712(21)00462-8/ fulltext. Acceso en: 20 out. 2021.

YU, Yuetian et al. A bibliometric analysis using VOSviewer of publications on COVID-19. Annals of Translational Medicine, Hong Kong, v. 8, n. 13, p. 816, 2020. Disponible en: https://atm.amegroups.com/ article/view/46197/html. Acceso en: 20 oct. 2021.

ZYOUD, Sa'ed H.; AL-JABI, Samah W. Mapping the situation of research on coronavirus disease-19 (COVID-19): a preliminary bibliometric analysis during the early stage of the outbreak. BMC Infectious Diseases, London, v. 20, n. 1, p. 561, 2020. DOI: https://doi.org/10.1186/s12879-020-05293-z. Disponible en: https://bmcinfectdis.biomedcentral.com/articles/10.1186/s12879-020-05293-z. Acceso en: 20 oct. 2021. 